\title{
Diskursanalyse und Diskurstheorie in der Kommunikationswissenschaft
}

\author{
Hans-Jürgen Bucher
}

Online publiziert: 30 . September 2020

(C) Der/die Autor(en) 2020

\section{Sammelrezension}

1. Wiedemann, Thomas/Lohmeier, Christine (Hrsg.): Diskursanalyse für die Kommunikationswissenschaft. Theorien Vorgehen, Erweiterungen. Wiesbaden: Springer VS 2019. 374 Seiten. Preis: $€ ~ 39,99$ (e-book)

2. Van Brussel, Leen/Carpentier, Nico/De Cleen, Benjamin (Hrsg.): Communication and discourse theory. Collected works of the Brussels Discourse Theory Group. Bristol: intellect 2019. 312 Seiten. Preis: $£ 24,00$ (e-book)

Vor dem Hintergrund einer Feststellung von Paddy Scannell in seinem Buch Media and Communication, ,that the question of communication was not central to the study of media in the twentieth century“, (2007, S. 3) ist es ein positives Zeichen, wenn in einem Jahr gleich zwei Sammelbände zur Diskursanalyse bzw. zur Diskurstheorie in der Medienforschung erscheinen. Diesen Sammelbänden ist gemeinsam, dass sie mit der Fokussierung auf diskursive Aspekte die sinn- und bedeutungskonstituierende Leistung der Medienkommunikation ins Zentrum stellen, deren Vernachlässigung Scannell beklagt. Dementsprechend sehen die HerausgeberInnen der beiden Bände in der Diskursanalyse und der Diskurstheorie übereinstimmend eine produktive Ergänzung der traditionellen Massenkommunikationsforschung insbesondere im Bereich der qualitativen Forschung. Es stellt sich deshalb die Frage, inwiefern sie diese Komplementärfunktion erfüllen, wo sie Erweiterungen kommunikationswissenschaftlicher Forschung präsentieren und welche Anschlussstellen sie dafür anbieten.

Prof. Dr. H.-J. Bucher $(\bowtie)$

Fachbereich II - Medienwissenschaft, Universität Trier, Universitätsring 15, 54296 Trier,

Deutschland

E-Mail: bucher@uni-trier.de 
Das Herausgeber-Team des Bandes „Diskursanalyse für die Kommunikationswissenschaft" will entsprechend seiner Einleitung diesen Anspruch auf drei Ebenen einlösen: Die Diskursanalyse soll als „Erkenntnisinstrument einer qualitativen Medienforschung theoretisch eingeordnet und reflektiert werden“, sie soll die Methodenkompetenz erweitern und sie soll drittens die „Anschlussfähigkeit der Kommunikationswissenschaft an große Fragen in der Gesellschaft erhöhen“" (S. 7/8). Zur Umsetzung des dritten Ziels beziehen sich die Beiträge nahezu ausschließlich auf das von Michel Foucault entwickelte Forschungsprogramm, das es erlaubt, mit Hilfe seines Begriffsinstrumentariums wie Dispositiv, Ordnung des Diskurses, Wissensordnung, Exklusion oder Inklusion, die Ebene der Diskurse auf gesellschaftliche Machtverhältnisse zu beziehen. Eine weitere Brücke zwischen Makro- und Mikroanalyse bildet die von Foucault inspirierte Wissenssoziologie, wie sie im Teil I ,Theoretische Positionen“ von Rainer Keller dargestellt wird. Angesichts der Tatsache, dass sich eine Forschungstradition zur epistemischen Leistung des Journalismus zu etablieren beginnt, ist die von Keller vorgestellte wissenssoziologische Diskursanalyse hochgradig anschlussfähig an kommunikationswissenschaftliche Fragestellungen.

Die theoretische Einordnung der Diskursanalyse als Erkenntnisinstrument, die Teil I leisten soll, fällt allerdings verglichen mit anderen Sammelbänden und Handbücher zum selben Thema (z.B. mit dem zweibändigen Handbuch ,Diskursforschung“ oder dem Routledge Handbook of Discourse Analysis) zu knapp und wenig innovativ aus: zum einen haben die Autoren Christian Pentzold und Margarete Jäger ihre spezifische Sicht der Diskursanalyse in den letzten Jahren bereits mehrfach und ausführlicher präsentiert und zum andern fehlen Traditionen der Diskursanalyse, die sich mit Aspekten und Formen von Diskursen befassen, wie sie gerade für die Medienkommunikation charakteristisch sind: multimodale Diskurstheorien, die die ganze Breite der Symbolsysteme der Medienkommunikation im Blick haben (Film, Foto, Grafik, Sound, Design etc.), Interaktionstheorien, die die Dynamik dialogischer Kommunikationsformen in Hörfunk (Hörerpartizipation), Fernsehen (Talkshows) und Tageszeitungen (Leserbriefe) aber insbesondere in den Online-Medien (soziale Medien, Anschlusskommunikationen) untersucht haben oder Genretheorien, die mit ihren Typologien die undifferenzierte Redeweise von „Mediendiskursen“ spezifizieren könnten. Das Fehlen dieser Differenzierungen des Diskursbegriffs auf theoretischer Ebne setzt sich im empirischen zweiten Teil des Bandes fort: selbst wenn Fernsehnachrichten untersucht werden (Karidi/Meyen) oder Online-Kommunikation (Sell/Linke) werden Diskurse auf Texte reduziert. Will die Diskursanalyse mehr sein als ein methodischer und modischer Ersatzbegriff für die ,,qualitative Inhaltsanalyse“" so müsste sie vor allem auf der Ebene der Gegenstandstheorie die genannten Differenzierungsvorschläge nutzen, um über eine Reduktion der Medienkommunikation auf „Texte“ hinauszugelangen. Werden Diskurse als ,Sinnproduktionsverfahren“ begriffen, so impliziert diese Ausrichtung auch die Integration der Rezeptionsperspektive, was immerhin in dem Beitrag von Johannes Angermuller mit dem Begriff des ,leserorientierten Konstruktivismus“ angesprochen ist.

Die empirischen Beiträge im Teil II und die Beiträge in Teil III, die Erweiterungen der Diskursanalyse vorschlagen, belegen, dass die diskursanalytische Perspektive für verschiedene Forschungsfelder der Medien- und Kommunikationswissenschaft produktiv sein kann: für die Journalismusforschung (Kirchhoff) oder die Online- 
Forschung, aber auch für die Entwicklung neuer Fragestellungen zur medialen Konstruktion von Identitäten (Lönnendonker), von Lebensweltkonzepten (Fingerling/ Godemann) oder zur Begriffsgeschichte politischer Kategorien (Rheindorf/Wodak).

Angesichts der Tatsache, dass es in der Deutschen Gesellschaft für Publizistikund Kommunikationswissenschaft seit 2007 eine Fachgruppe „Mediensprache - Mediendiskurse“ gibt, mit regelmäßigen Tagungen, Publikationen und Forschungsprojekten ist es allerdings eine erstaunliche Behauptung des Herausgeber-Teams, dass „die diskursanalytische Herangehensweise in der deutschsprachigen Kommunikationswissenschaft und Medienforschung noch immer eine Seltenheit [ist]“ (S. 1).

Der Band „Communication and Discourse Theory“ herausgegeben von der in der deutschen Kommunikationswissenschaft kaum rezipierten „Brussels Discourse Theory Group“ hat mit dem Band von Wiedemann und Lohmeier zwei Gemeinsamkeiten: die Sichtweise von Mediendiskursen als Instanzen der Bedeutungs- und Sinnkonstitution (,Signifying machines“) und die Intention, eine gesellschaftskritische Diskursanalyse zu entwickeln, die anschlussfähig ist an gesellschaftspolitisch relevante Problemstellungen. Stärker als der Bezug auf Foucault dienen die Arbeiten von Ernesto Laclau und Chantal Mouffe - insbesondere Hegemony and socialist strategy: towards a radical democratic politics - als theoretische Referenz. Deren antagonistische Gesellschafts- und Diskurskonzeption spiegelt sich in den Beiträgen des Bandes einerseits in der Begrifflichkeit zur Analyse diskursiver Hegemonie wie „Subjektposition“, ,Artikulation“ oder ,nodal points“ (Bedeutungsverfestigungen). Andererseits befassen sich alle Beiträge mit gesellschaftspolitischen Kontroversen, in denen um politisch-ideologische Dominanz gerungen wird, z. B. um die Auslegung der Kategorien „Populärkultur“ und „Volk“ in einer Debatte zwischen der rechtsextremen Partei Vlaams Belang und den Veranstaltern eines gegenkulturellen Rockkonzerts (De Cleen/Carpentier), um die Idee von Feminismus, die die schwedische „Feministik initiativ“ in der Wahlkampfkommunikation zu etablieren versucht (Filimonov/Svensson), um die Deutung der Sterbehilfe in der Zeitungsberichterstattung (van Brussel) oder um das Konzept der ehelichen Treue und die mediale Instrumentalisierung von teilnehmenden Paaren in der Anschlusskommunikation zur Reality-Verführungssendung Temptation Island. Es sind insbesondere die beiden Abschnitte im Band „Production“ und „Audiences and Participation“ die Anschlussstellen für Journalismus- und Rezeptionsforschung aufzeigen. Im Unterschied zur Verdinglichung des Diskurses als ,übersubjektive epistemische Ordnungen“ (S. 20) im deutschen Sammelband betonen die Vertreter des Brüsseler Diskurskonzeptes deutlich stärker dessen dynamische und agonistische Aspekte und positionieren Diskurse dementsprechend, in a middle position between structure and agency“ (S. 6).

Auffallend ist in beiden Sammelbänden die Gegenstandsferne der präsentierten Analysen, die sich dementsprechend streckenweise eher wie Leitartikel zum entsprechenden Thema oder Paraphrasierungen der untersuchten Medieninhalten lesen und weniger als Empirie-gesättigte Interpretationen des entsprechenden Materials. Dennoch liegt der Verdienst der beiden Sammelbände darin, die symbol- und zeichenbasierten Aspekte der Medienkommunikation in den Fokus der Forschung gerückt zu haben, ohne dadurch den Bezug zu gesellschaftspolitisch relevanten Fragestellungen aufzugeben. Dieses Potential ließe sich durch die Integration bislang vernachlässig- 
ter diskursanalytischer Tradition ausbauen, auch wenn das auf der Theorieebene zu einer noch stärkeren Ausdifferenzierung des Diskursbegriffs führen wird.

Funding Open Access funding enabled and organized by Projekt DEAL.

Open Access Dieser Artikel wird unter der Creative Commons Namensnennung 4.0 International Lizenz veröffentlicht, welche die Nutzung, Vervielfältigung, Bearbeitung, Verbreitung und Wiedergabe in jeglichem Medium und Format erlaubt, sofern Sie den/die ursprünglichen Autor(en) und die Quelle ordnungsgemäß nennen, einen Link zur Creative Commons Lizenz beifügen und angeben, ob Änderungen vorgenommen wurden.

Die in diesem Artikel enthaltenen Bilder und sonstiges Drittmaterial unterliegen ebenfalls der genannten Creative Commons Lizenz, sofern sich aus der Abbildungslegende nichts anderes ergibt. Sofern das betreffende Material nicht unter der genannten Creative Commons Lizenz steht und die betreffende Handlung nicht nach gesetzlichen Vorschriften erlaubt ist, ist für die oben aufgeführten Weiterverwendungen des Materials die Einwilligung des jeweiligen Rechteinhabers einzuholen.

Weitere Details zur Lizenz entnehmen Sie bitte der Lizenzinformation auf http://creativecommons.org/ licenses/by/4.0/deed.de.

Prof. Dr. Hans-Jürgen Bucher ist em. Professor für Medienwissenschaft an der Universität Trier. 